\title{
Human severe sepsis cytokine mixture increases $\beta 2$-integrin-dependent polymorphonuclear leukocyte adhesion to cerebral microvascular endothelial cells in vitro
}

\author{
Chris Blom ${ }^{1}$, Brittany L Deller ${ }^{1}$, Douglas D Fraser ${ }^{1,2,3,4,5}$, Eric K Patterson ${ }^{3}$, Claudio M Martinn, 3, Bryan Young ${ }^{5}$, \\ Patricia C Liaw ${ }^{7,8}$, Payam Yazdan-Ashoori ${ }^{1}$, Angelica Ortiz ${ }^{2}$, Brian Webb ${ }^{9}$, Greg Kilmer ${ }^{9}$, David E Carter ${ }^{10}$ \\ and Gediminas Cepinskas ${ }^{3,11^{*}}$
}

\begin{abstract}
Introduction: Sepsis-associated encephalopathy (SAE) is a state of acute brain dysfunction in response to a systemic infection. We propose that systemic inflammation during sepsis causes increased adhesion of leukocytes to the brain microvasculature, resulting in blood-brain barrier dysfunction. Thus, our objectives were to measure inflammatory analytes in plasma of severe sepsis patients to create an experimental cytokine mixture (CM), and to use this CM to investigate the activation and interactions of polymorphonuclear leukocytes (PMN) and human cerebrovascular endothelial cells (hCMEC/D3) in vitro.

Methods: The concentrations of 41 inflammatory analytes were quantified in plasma obtained from 20 severe sepsis patients and 20 age- and sex-matched healthy controls employing an antibody microarray. Two CMs were prepared to mimic severe sepsis (SSCM) and control (CCM), and these CMs were then used for PMN and hCMEC/D3 stimulation in vitro. PMN adhesion to hCMEC/D3 was assessed under conditions of flow (shear stress $0.7 \mathrm{dyn} / \mathrm{cm}^{2}$ ).

Results: Eight inflammatory analytes elevated in plasma obtained from severe sepsis patients were used to prepare SSCM and CCM. Stimulation of PMN with SSCM led to a marked increase in PMN adhesion to hCMEC/D3, as compared to CCM. PMN adhesion was abolished with neutralizing antibodies to either $\beta 2$ (CD18), $a_{L} / \beta_{2}$ (CD11a/CD18; LFA-1) or $a_{M} / \beta_{2}$ (CD11 $\beta / C D 18 ;$ Mac-1) integrins. In addition, immune-neutralization of the endothelial (hCMEC/D3) cell adhesion molecule, ICAM-1 (CD54) also suppressed PMN adhesion.
\end{abstract}

Conclusions: Human SSCM up-regulates PMN pro-adhesive phenotype and promotes PMN adhesion to cerebrovascular endothelial cells through a $\beta 2$-integrin-ICAM-1-dependent mechanism. PMN adhesion to the brain microvasculature may contribute to SAE.

\section{Introduction}

Sepsis, a systemic inflammatory response to a known or suspected infection, is a leading cause of admission and mortality in intensive care units (ICUs) [1]. Severe sepsis refers to sepsis accompanied by acute organ dysfunction [2]. Up to $70 \%$ of patients with severe sepsis experience an acute

\footnotetext{
* Correspondence: gcepinsk@uwo.ca

${ }^{3}$ Centre for Critical IIIness Research, Lawson Health Research Institute,

800 Commissioners Rd East, London, ON N6C 6B5, Canada

${ }^{11}$ Department of Medical Biophysics, Western University, 1151 Richmond Str.

North, London, ON N6A 5C1, Canada

Full list of author information is available at the end of the article
}

neurological dysfunction known as sepsis-associated encephalopathy (SAE) [3]. Acute brain dysfunction in severe sepsis patients is associated with increased rates of mortality and long-term cognitive impairment in survivors $[4,5]$.

The mechanisms of SAE are unknown, but possible instigating factors may be the early activation of the innate immune response and microcirculatory dysfunction that are common manifestations of severe sepsis [6,7]. Activated leukocytes and vascular endothelial cells contribute to systemically increased concentrations of cytokines and chemokines in patients with sepsis [8].

\section{Biomed Central}

(c) 2015 Blom et al.; licensee BioMed Central. This is an Open Access article distributed under the terms of the Creative Commons Attribution License (http://creativecommons.org/licenses/by/4.0), which permits unrestricted use, distribution, and reproduction in any medium, provided the original work is properly credited. The Creative Commons Public Domain Dedication waiver (http://creativecommons.org/publicdomain/zero/1.0/) applies to the data made available in this article unless otherwise stated. 
Circulating inflammatory mediator(s) activate polymorphonuclear leukocytes (PMN), leading to up-regulation of the pro-adhesive phenotype and recruitment of PMN to the inflamed tissues and/or organs $[9,10]$. While PMN are critical for the host defense against pathogens, the systemic activation of PMN also correlates with organ dysfunction in septic patients [11]. Animal models of sepsis demonstrate that overwhelming PMN recruitment is associated with dysfunction of the affected organs, including the brain [12-14].

Leukocyte (such as PMN) recruitment to the microvasculature can be broken down to two general steps: rolling and adhesion $[9,15]$. Interactions between adhesion molecules expressed constitutively or in response to inflammation, by both endothelial cells and leukocytes, are responsible for cellular interactions. PMN rolling is primarily controlled by a family of glycoproteins (selectins) expressed on both PMN (L-selectin) and endothelial cells (such as P- and E-selectins). Subsequently, PMN firm adhesion is mediated primarily through $\beta-2$ integrin (CD18; expressed by PMN) and ICAM-1 (CD54; expressed by endothelial cells) interaction. The $\beta 2$ integrins form heterodimers with either integrin- $\alpha \mathrm{L}$ (CD11a) or integrin- $\alpha M$ (CD11b) to form $\alpha_{L} / \beta_{2}$ (CD11a/ CD18; LFA-1) or $\alpha_{M} / \beta_{2}$ (CD11b/CD18; Mac-1), respectively. Both LFA-1 and Mac-1 interact with ICAM-1 to promote PMN adhesion to endothelial cells.

Endothelial cells of the blood-brain barrier (BBB) are likely disrupted during SAE, as vasogenic edema is consistently found on head magnetic resonance imaging in sepsis [16]. Lesions in the subcortical white matter, also consistent with vasogenic edema, are particularly prominent in cases of fatal septic shock [17]. Increased signal associated with contrast agent administration during SAE indicates BBB disruption [6]. The mechanism(s) of BBB disruption in sepsis, however, remain poorly understood.

In this study, we hypothesized that the inflammation associated with severe sepsis would result in greater PMN interactions with the brain microvasculature. Thus, we first aimed to measure circulating inflammatory mediators in blood plasma from patients with severe sepsis, and then investigate the effects of these inflammatory analytes on the pro-adhesive phenotype of human PMNs and human cerebrovascular endothelial cells (hCMEC/D3) in vitro.

\section{Materials and methods}

The institutional review boards of Hamilton Health Sciences (Hamilton, ON, Canada) and the University of Western Ontario (London, ON, Canada) approved this study. Consent was obtained from the ICU patients or their legal guardians. Consistency of patient blood sampling in the participating centers was monitored by a study investigator (PCL).

\section{Severe sepsis patients and blood plasma}

Blood plasma collection was described previously [18]. Briefly, blood was collected from ICU patients within the first 24 hours of severe sepsis diagnosis according to the American College of Chest Physicians/Society of Critical Care Medicine (ACCP/SCCM) guidelines $[2,19]$. Venous blood $(4.5 \mathrm{~mL}$ ) was drawn via indwelling catheters (severe sepsis patients) or fresh venipuncture (from age- and sex-matched healthy controls), and immediately transferred into $15 \mathrm{~mL}$ polypropylene tubes (VWR, Mississauga, ON, Canada) containing $0.5 \mathrm{~mL}$ of $0.105 \mathrm{~mol} / \mathrm{L}$ buffered trisodium citrate (pH 5.4) (Sigma-Aldrich, St. Louis, MO, USA) with $100 \mu \mathrm{L}$ of $1 \mathrm{~mol} / \mathrm{L}$ benzamidine $\mathrm{HCl}$ (approximately $20 \mathrm{mmol} / \mathrm{L}$ benzamidine), a serine protease inhibitor (Sigma-Aldrich, St. Louis, MO, USA). The blood was immediately centrifuged at $1,500 \mathrm{~g}$ for 10 minutes at $20^{\circ} \mathrm{C}$, and the plasma was stored in aliquots at $-80^{\circ} \mathrm{C}$. Care was taken to ensure that freeze/thaw cycles were avoided. Baseline characteristics of severe sepsis patients were recorded at ICU admission (Table 1). Baseline

Table 1 Baseline characteristics of 20 patients with severe sepsis on ICU day 1

\begin{tabular}{|c|c|}
\hline Age, years & $59 \pm 4(20-80)$ \\
\hline Gender, female & $6(30 \%)$ \\
\hline APACHE II score & $25.8 \pm 2.1(6-42)$ \\
\hline MOD score & $10.4 \pm 0.86(3-15)$ \\
\hline Absolute neutrophil count (ANC) $)^{a}$ & $11.5 \pm 0.9(3.7-19.5)$ \\
\hline \multicolumn{2}{|l|}{ Co-morbidities, number (\% of total) } \\
\hline Cardiovascular & $9(45 \%)$ \\
\hline Pulmonary & $5(25 \%)$ \\
\hline Hepatitis/pancreatitis & $5(25 \%)$ \\
\hline Inflammatory & $3(15 \%)$ \\
\hline Neurological & $3(15 \%)$ \\
\hline Renal & $3(15 \%)$ \\
\hline Diabetes & $2(10 \%)$ \\
\hline \multicolumn{2}{|c|}{ Primary site of infection, number (\% of total) } \\
\hline Lung & $11(55 \%)$ \\
\hline Abdomen & $3(15 \%)$ \\
\hline Blood & $1(5 \%)$ \\
\hline Urinary Tract & $0(0 \%)$ \\
\hline Other & $4(20 \%)$ \\
\hline Unknown & $1(5 \%)$ \\
\hline \multicolumn{2}{|c|}{ Total number of positive cultures (\% of total) ${ }^{b}$} \\
\hline Gram-negative bacteria & $10(50 \%)$ \\
\hline Gram-positive bacteria & $9(45 \%)$ \\
\hline Fungus & $2(10 \%)$ \\
\hline
\end{tabular}

Data are presented as mean \pm SE (range) or $n(\%) .{ }^{a}$ Normal ANC $=1.5-8.0 .{ }^{b}$ Includes blood and non-blood cultures, therefore the total is $>100 \%$. Four poly-microbial non-blood cultures were not included in the table. APACHE II, Acute Physiology and Chronic Health Evaluation II; MOD, Multiple organ dysfunction. 
characteristics included demographics, clinical predictive scores (Acute Physiology and Chronic Health Evaluation II (APACHE II) and multiple organ dysfunction syndrome (MODS)), site of infection, positive blood cultures, and co-morbidities.

\section{Antibody microarray}

Sample analysis was performed by an investigator blinded to the experimental cohort (severe sepsis versus healthy control) in one laboratory (DDF) as batched samples [18]. A total of 4 antibody microarrays were used to analyze the concentrations of 41 different inflammatory mediators in 20 plasma samples from both severe sepsis patients and age- and sex-matched healthy controls. Plasma samples were each diluted 1:3 in Array Sample Diluent and were run on a Thermo Scientific ExcelArray $^{\text {mit }}$ (Rockford, IL, USA): Inflammation I (product number 82002), Inflammation II (product number: 82003), Chemotaxis (product number: 82006), and Angiogenesis (product number: 82004) [20].

Protein concentrations were determined for the following human analytes: angiogenin, basic fibroblast growth factor (FGF $\beta$ ), cysteine-cysteine chemokine ligand 1 (CCL1; I-309), eotaxin, epidermal growth factor (EGF), fas ligand (FasL), granulocyte colony-stimulating factor (G-CSF), granulocyte-macrophage colony-stimulating factor (GM$\mathrm{CSF})$, growth-regulated oncogene alpha (GRO- $\alpha$ ), heparinbinding epidermal growth factor (HB-EGF), hepatocyte growth factor (HGF), interferon gamma (IFNY), interferoninducible protein $10 \mathrm{kDa}$ (IP-10), interleukin-1 alpha (IL-1 $\alpha$ ), interleukin-1 beta (IL-1 $\beta$ ), interleukin-2 (IL-2), interleukin-3 (IL-3), interleukin-4 (IL-4), interleukin-5 (IL-5), interleukin-6 (IL-6), interleukin-7 (IL-7), interleukin8 (IL-8), interleukin-10 (IL-10), interleukin-12 (IL-12), interleukin-13 (IL-13), interleukin-15 (IL-15), interleukin17 (IL-17), keratinocyte growth factor (KGF), macrophagederived chemokine (MDC), macrophage inflammatory protein-1 alpha (MIP-1 $\alpha)$, macrophage inflammatory protein-1 beta (MIP-1 $\beta$ ), monocyte chemotactic protein-1 (MCP-1), monocyte chemotactic protein-3 (MCP-3), neutrophil-activating protein-2 (NAP-2), normal T-cell expressed and secreted (TARC), placenta growth factor (P1GF), regulated upon activation, normal T cell expressed and secreted (RANTES), tissue inhibitor of metalloproteinase 1 (TIMP-1), tumor necrosis factor alpha (TNF- $\alpha$ ), tumor necrosis factor beta (TNF- $\beta$ ), and vascular endothelial growth factor (VEGF).

Briefly, $100 \mu \mathrm{L}$ of multiplexed standards (1,000-12.3 $\mathrm{pg} / \mathrm{mL}$ ) or diluted plasma was applied to each well and incubated at room temperature on a shaker at $200 \mathrm{rpm}$ for two hours. After sample incubation, the microarrays were rinsed three times with wash buffer. Prior to reincubation on a shaker for one hour, $75 \mu \mathrm{L}$ pre-titered biotinylated detector antibodies were added to each well.
Microarrays were washed three times and $100 \mu \mathrm{L}$ Streptavidin DyLight 649 (part of Thermo Scientific ExcelArray ${ }^{\mathrm{Tm}}$ kit; Thermo Fisher Scientific, Rockford, IL, USA) was applied to each well, preceding a final incubation of 30 minutes on a shaker. Microarrays were washed five times and dipped briefly in a final rinse solution. Slides were then centrifuged at approximately 200 g until dry. Methodologies for microarray production and antibody cross reactivity assays have been described previously [20]. Cross reactivity on the microarray antibody pairs is $\leq 5 \%$, as reported by the manufacturer. Microarrays were imaged using an Alphascan $^{\mathrm{Tm}}$ Microarray Imager (Alpha Innotech, San Leandro, CA, USA) and spot densitometry was performed by Thermo Fisher (Rockford, IL, USA) using ArrayVision ${ }^{\text {Tw }}$ Software (GE Healthcare, Piscataway, NJ, USA).

\section{Human cytokine mixtures}

The most up-regulated $(P<0.001)$ human analytes detected in the plasma of severe sepsis patients (IL-8, G-CSF, IP-10, GRO- $\alpha$, HGF, MIP-1 $\beta$, and MCP-1; Table 2) were selected to make the severe sepsis cytokine mixture (SSCM) and control cytokine mixture (CCM). The sepsis-relevant cytokine IL-6 [21,22] was also significantly up-regulated in the plasma of severe sepsis patients $(P=0.017)$ and was added to each cytokine mixture. Recombinant proteins were suspended in PBS containing 1\% BSA at 100X concentration measured in severe sepsis blood plasma or healthy control blood plasma (Table 2). Each cytokine mixture was stored at $-20^{\circ} \mathrm{C}$ until thawed for experiments. To treat cells, each cytokine mixture was diluted at $100 \mathrm{X}$ concentration in either PBS or VascuLife EnGS-Mv (LifeLine cell technology, Walkersville, MD, USA) cell culture medium to the corresponding analyte concentrations measured in control blood

\begin{tabular}{|c|c|c|c|c|}
\hline Analyte & Control & Severe sepsis & Fold increase & $P$ value \\
\hline$\overline{\mathrm{MCP}-1}$ & $41.8 \pm 12.1$ & $1067.8 \pm 196.4$ & 25 & $<0.001$ \\
\hline G-CSF & $29.3 \pm 9.5$ & $938.4 \pm 278.5$ & 32 & $<0.001$ \\
\hline IL-8 & $10.0 \pm 0.0$ & $211.9 \pm 43.4$ & 21 & $<0.001$ \\
\hline HGF & $290.3 \pm 60.8$ & $3346.8 \pm 570.5$ & 12 & $<0.001$ \\
\hline MIP-1 $\beta$ & $49.7 \pm 12.1$ & $381.1 \pm 116.0$ & 8 & $<0.001$ \\
\hline $\mid \mathrm{P}-10$ & $214.4 \pm 26.8$ & $1552.0 \pm 254.5$ & 7 & $<0.001$ \\
\hline GROa & $92.5 \pm 24.2$ & $637.5 \pm 136.3$ & 7 & $<0.001$ \\
\hline $\mathbb{I L}-6$ & $80.8 \pm 31.8$ & $387.6 \pm 158.4$ & 5 & 0.017 \\
\hline
\end{tabular}

Plasma was obtained from severe sepsis patients and compared to plasma from age- and sex-matched healthy controls. Analytes were measured with antibody

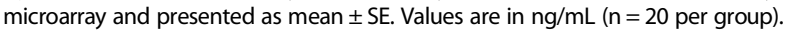
MCP-1-monocyte chemotactic protein-1; GCSF-granulocyte colony-stimulating factor; IL-8- interleukin-8; HGF- hepatocyte growth factor; MIP-1 $\beta$ - macrophage inflammatory protein-1 beta; IP-10- interferon-inducible protein $10 \mathrm{kDa}$; GRO-a- growth-regulated oncogene alpha; IL-6- interleukin-6. 
plasma or severe sepsis blood plasma, respectively. All human recombinant analytes were obtained from either Ebioscience (San Diego, CA, USA) or Invitrogen (Carlsbad, CA, USA).

\section{Human cerebral microvascular endothelial cells}

hCMECs (hCMEC/D3 cell line) were isolated and immortalized by Dr Pierre-Olivier Couraud and his colleagues (INSERM, Paris, France; [23]). The immortalization of CMEC was performed via sequential lentiviral transduction of hTERT and SV40 large T antigen transduction into a primary culture of adult CMEC. The hCMEC/D3 cell line offers a unique opportunity to study human cerebrovascular cells in isolation from other cells, and represents a stable, fully characterized, and well-differentiated line of CMEC.

hCMEC/D3 were grown at $37^{\circ} \mathrm{C}$ and $5 \% \mathrm{CO}_{2}$ in VascuLife EnGS-Mv (LifeLine Cell Technology, Walkersville, MD, USA) cell culture medium supplemented with recombinant human EGF (0.2\%), EnGS (5 ng/mL), heparin $(0.75 \mathrm{U} / \mathrm{mL})$, fetal bovine serum ((FBS) 5\%), ascorbic acid $(50 \mu \mathrm{g} / \mathrm{mL})$, L-glutamine $(10 \mathrm{mM})$, and hydrocortisone $(1.0 \mu \mathrm{g} / \mathrm{mL})$ (LifeLine Cell Technology, Walkersville, MD, USA) [24]. Penicillin $(100 \mathrm{IU} / \mathrm{mL})$ and streptomycin $(100 \mu \mathrm{g} / \mathrm{mL})$ (Wisent Inc, St Bruno, QC, Canada) were also added to cell culture media. hCMEC/D3 at passage 35-50 were used in all experiments.

\section{Human polymorphonuclear leukocyte isolation}

Human PMN were isolated from venous blood of healthy adults immediately before experiments as previously described by us [24]. Briefly, heparinized whole blood (10 mL; $10 \mathrm{U} / \mathrm{mL}$; Pharmaceutical Partners of Canada, Richmond Hill, ON, Canada) was gently mixed by inversion (10 times) with $5 \mathrm{~mL}$ of $3 \%$ dextran in PBS ( $\mathrm{pH}$ 7.4). Sedimentation of red blood cells was performed via incubation of the mixture for 20 minutes at $23^{\circ} \mathrm{C}$. Blood plasma was removed and gently added onto $5 \mathrm{~mL}$ of Histopaque lymphocyte separation medium (density of $1.077 \mathrm{~g} / \mathrm{mL}$; Sigma-Aldrich, St Louis, MO, USA). Samples were centrifuged for 30 minutes at $400 \times \mathrm{g}$ at $23^{\circ} \mathrm{C}$. Following centrifugation, the pellet (PMN and remaining red blood cells) was re-suspended in $15 \mathrm{~mL}$ cold red blood cell lysis buffer, containing $\mathrm{NH} 4 \mathrm{Cl}(8.3 \mathrm{~g} / \mathrm{L})$, KHCO3 $(1.0 \mathrm{~g} / \mathrm{L})$, and EDTA $(0.14 \mathrm{mM})$ (all purchased from Sigma-Aldrich, St. Louis, MO, USA) in water, adjusted to a pH of 7.2. After five minutes incubation at $4^{\circ} \mathrm{C}$, mixtures were centrifuged at $300 \times \mathrm{g}$ for five minutes. PMN were re-suspended in $1 \mathrm{~mL}$ PBS $\left(4^{\circ} \mathrm{C}\right)$, stored on ice until use, and counted using a hemocytometer (VWR, Mississauga, ON, Canada). This procedure yields a PMN population that is 95 to $98 \%$ viable (as measured by trypan blue exclusion) and $98 \%$ pure (as measured by acetic acid-crystal violet staining) (both reagents were purchased from Sigma-Aldrich, St. Louis, MO, USA).

\section{Polymorphonuclear leukocyte adhesion to hCMEC/D3}

hCMEC/D3 were grown to confluence in $\mu$-Slide $\mathrm{VI}^{0.4}$ parallel flow channels (ibidi; MOFA Global, Ingersoll, $\mathrm{ON}$, Canada) and interacted with PMN as previously described by us $[24,25]$. Briefly, hCMEC/D3 were seeded at a density of 11,000 cells per channel and grown for 5 days, followed by 5 hours of stimulation with either CCM or SSCM diluted in serum free-VascuLife EnGSMv cell culture medium. The CCM and SSCM treatments did not affect hCMEC/D3 viability as assessed by ViaCount Flex (Cedarlane, Burlington, ON, Canada) assay and flow-cytometry approach (data not shown). Each channel was then perfused (that is, washed) in the presence of laminar shear stress of $0.7 \mathrm{dyn} / \mathrm{cm}^{2}$ for one minute with VascuLife EnGS-Mv cell culture medium, using a Harvard Apparatus 22-syringe pump (Instech, Plymouth Meeting, PA, USA). Subsequently and continuously, hCMEC/D3 were perfused with freshly isolated PMN $\left(1 \times 10^{6} \mathrm{PMN} / \mathrm{mL}\right.$; suspended in serum freeVascuLife EnGS-Mv cell culture medium) that have been stimulated either with CCM or SSCM for 10 minutes. The experiment was visualized and video recorded using a Nikon DIAPHOT-300 inverted microscope (Nikon, Chiyoda-ku, Tokyo, Japan) equipped with a temperature-controlled chamber $\left(37^{\circ} \mathrm{C}\right)$ and Panasonic WJ-810 video-camera (Panasonic, Kadoma, Osaka, Japan) connected to an SV-video recorder (Panasonic). For each experiment, 5 random fields of view $\left(0.1 \mathrm{~mm}^{2}\right.$ each) were recorded for 30 seconds, starting at 4 minutes after initiation of the PMN perfusion. The recorded PMN-hCMEC/ D3 adhesive interactions were video-analyzed to count the number of firmly adherent PMN (stationary for at least five seconds). The average number of adherent PMN was expressed as PMN/0.1 $\mathrm{mm}^{2}$.

\section{Adhesion molecule immuno-neutralization}

PMN $\left(2 \times 10^{7} / \mathrm{mL}\right)$ suspended in PBS were stimulated with CCM or SSCM at $23^{\circ} \mathrm{C}$ for 10 minutes in a total volume of $0.2 \mathrm{~mL}$. Subsequently, function neutralizing monoclonal antibodies (all IgG1א subclass; BioLegend, San Diego, CA, USA) directed against either human $\beta_{2}$-integrin (clone TS $1 / 18 ; 25 \mu \mathrm{g} / \mathrm{mL}$ ), human LFA-1 (clone HI111; $5 \mu \mathrm{g} / \mathrm{mL}$ ), or Mac-1 (clone ICRF44; $5 \mu \mathrm{g} / \mathrm{mL}$ ), were added to the PMN suspension for an additional $15 \mathrm{mi}-$ nutes. An isotype control (IgG1א; BioLegend, San Diego, CA, USA) antibody was used in parallel experiments at the corresponding concentrations. Following treatment, PMN were immediately added to VascuLife EnGS-Mv cell culture medium to a final concentration of $1 \times 10^{6} / \mathrm{mL}$ and perfused over hCMEC/D3. 
In some experiments, hCMEC/D3 grown in laminar flow micro channels (ibidi $\mathrm{GmbH}$ ) were stimulated with either CCM or SSCM and subsequently treated with function neutralizing anti-human ICAM-1 (CD54; clone HCD54; $5 \mu \mathrm{g} / \mathrm{mL}$ ) or isotype control (both IgG1k subclass; BioLegend, San Diego, CA, USA) antibodies in VascuLife EnGS-Mv cell culture medium for 30 minutes at $37^{\circ} \mathrm{C}$, preceding $\mathrm{PMN}$ perfusion.

\section{RNA isolation, CDNA synthesis and quantitative PCR}

hCMEC/D3 were seeded (500,000 per well) on six-well plates and grown for two days, preceding four hours of stimulation with either CCM or SSCM. Following stimulation, hCMEC/D3 were washed three times with PBS and RNA was extracted with $1 \mathrm{~mL} \mathrm{TRIzol}^{\circ}$ reagent (Life Technologies, Carlsbad, CA, USA), as per the manufacturer's protocol except the RNA pellet was washed three times with $75 \%$ ethanol. RNA was resuspended in $20 \mu \mathrm{L}$ of $10 \mathrm{mM}$ Tris, $\mathrm{pH}$. The purity and concentration of RNA was determined using a Biophotometer (Eppendorf, Hamburg, Germany). One microgram of RNA per sample was reverse transcribed using iScript $^{\text {tm }}$ Reverse Transcription Supermix (Bio-Rad, Hercules CA, USA) according to the manufacturer's protocol.

Quantitative PCR (qPCR) was performed using Sso Fast $^{\text {tw }}$ Probes Supermix (Bio-Rad, Hercules, CA, USA), according to the manufacturer's protocol, using cDNA at concentrations previously determined to be within 90 to 110\% efficiency. Primers were predesigned (Applied Biosystems, Foster City, CA, USA): 185 (Hs99999901_s1), glyceraldehyde-3-phosphate dehydrogenase (GAPDH; Hs99999905_m1), ICAM-1 (Hs00164932_m1), and VCAM-1 (Hs01003372_m1). Real-time PCR was run using a $\mathrm{C}^{1000}{ }^{\mathrm{ma}}$ Touch Thermal Cycler with CFX96 ${ }^{\mathrm{ma}}$ Real-Time System (Bio-Rad, Hercules CA, USA). Cycling conditions included one-time enzyme activation at $95^{\circ} \mathrm{C}$ for 30 seconds, followed by 50 cycles of denaturation at $95^{\circ} \mathrm{C}$ for 5 seconds, and annealing and extension at $60^{\circ} \mathrm{C}$ for 15 seconds. Target gene expression was normalized to GAPDH and $18 S$ and was expressed relative to gene expression by hCMEC/D3 treated with serum-free VascuLife EnGS-Mv cell culture media.

\section{Statistical analysis}

Data were analyzed using SigmaStat 3.5 (Systat Software Inc., San Jose, CA, USA). Continuous variables are reported with mean \pm SE. Groups were pre-screened for normality and compared with either a Student's t test or Mann-Whitney $U$ test. Multiple groups were analyzed using one-way analysis of variance (ANOVA) with Tukey's post-hoc test. A $P<0.05$ was considered statistically significant.

\section{Results}

Patient characteristics and plasma inflammatory biomarkers

The baseline characteristics at ICU admission of the 20 severe sepsis patients from whom blood plasma was collected are presented in Table 1 . Of these 20 patients with severe sepsis, 18 had septic shock (90\%) and 4 died (20\%). Antibody microarray analysis of plasma from severe sepsis and control patients indicate that 13 out of the 41 analytes measured were significantly up-regulated in severe sepsis. The measured concentrations of eight analytes were used to create the cytokine mixtures, both SSCM and CCM (IL-8, G-CSF, IP-10, GRO- $\alpha$, HGF, MIP-1 $\beta$, MCP-1, IL-6; Table 2; $\mathrm{n}=20$ per group). In addition, FGF $\beta(802.3 \pm 402.9$ versus $1,573.4 \pm 456.9 \mathrm{ng} /$ $\mathrm{mL} ; P<0.012)$, IL-15 (38.6 \pm 23.1 versus $256.1 \pm 128.7 \mathrm{ng} /$ $\mathrm{mL} ; P<0.031)$, MCP-3 $(22.4 \pm 4.6$ versus $93.9 \pm 33.0 \mathrm{ng} / \mathrm{mL}$; $P<0.011)$, HB-EGF $(21.2 \pm 7.8$ versus $642.0 \pm 254.4 \mathrm{ng} / \mathrm{mL}$; $P<0.044)$, and KGF $(33.7 \pm 9.8$ versus $177.1 \pm 103.0 \mathrm{ng} / \mathrm{mL}$; $P<0.046)$ were also significantly up-regulated in the plasma of severe sepsis patients, but not included in the cytokine mixtures.

\section{Polymorphonuclear leukocyte adhesion to hCMEC/D3}

$\mathrm{PMN}$ and/or hCMEC/D3 were stimulated with either SSCM or CCM and assessed for PMN adhesion to hCMEC/D3 in the presence of flow (laminar shear stress $0.7 \mathrm{dyn} / \mathrm{cm}^{2}$ ). Stimulation of hCMEC/D3 with SSCM did not significantly increase the adhesion of naïve (unstimulated) PMN (Figure 1). On the contrary, stimulation of PMN with SSCM significantly increased PMN adhesion to hCMEC/D3 $(P<0.05 ; \mathrm{n}=7$; Figure 1$)$, while simultaneous treatment of PMN and hCMEC/D3 with SSCM (co-stimulation) had no additive effects.

\section{Role of $\beta 2$-integrins and ICAM-1 in polymorphonuclear leukocyte adhesion to hCMEC/D3}

Antibody neutralization was used to assess the role of $\beta 2$-integrins on SSCM-induced PMN adhesion to hCMEC/D3. SSCM-stimulated PMN were treated with either a function neutralizing anti- $\beta_{2}$-integrin antibody or isotype control antibody before hCMEC/D3 interaction. Anti- $\beta_{2}$-integrin antibody, but not isotype matching control antibody, prevented PMN adhesion to naive (unstimulated) hCMEC/D3 $(P<0.05 ; \mathrm{n}=4$; Figure $2 \mathrm{~A})$. The anti- $\beta_{2}$-integrin antibody was also effective in preventing SSCM-induced PMN adhesion to SSCM-stimulated hCMEC/D3 $(P<0.05$; $\mathrm{n}=4$; Figure $2 \mathrm{~B}$ ). Finally, neutralizing antibodies revealed that both $\alpha_{L} / \beta_{2}(C D 11 a / C D 18 ; L F A-1)$ and $\alpha_{M} / \beta_{2}$ (CD11b/CD18; Mac-1) integrins equally contribute to SSCM-induced PMN adhesion to hCMEC/D3 $(P<0.05$; $\mathrm{n}=4$; Figure 3).

Interfering with hCMEC/D3 ICAM-1 function by pretreatment of hCMEC/D3 with an anti-ICAM-1 neutralizing antibody was also effective in reducing adhesion of 


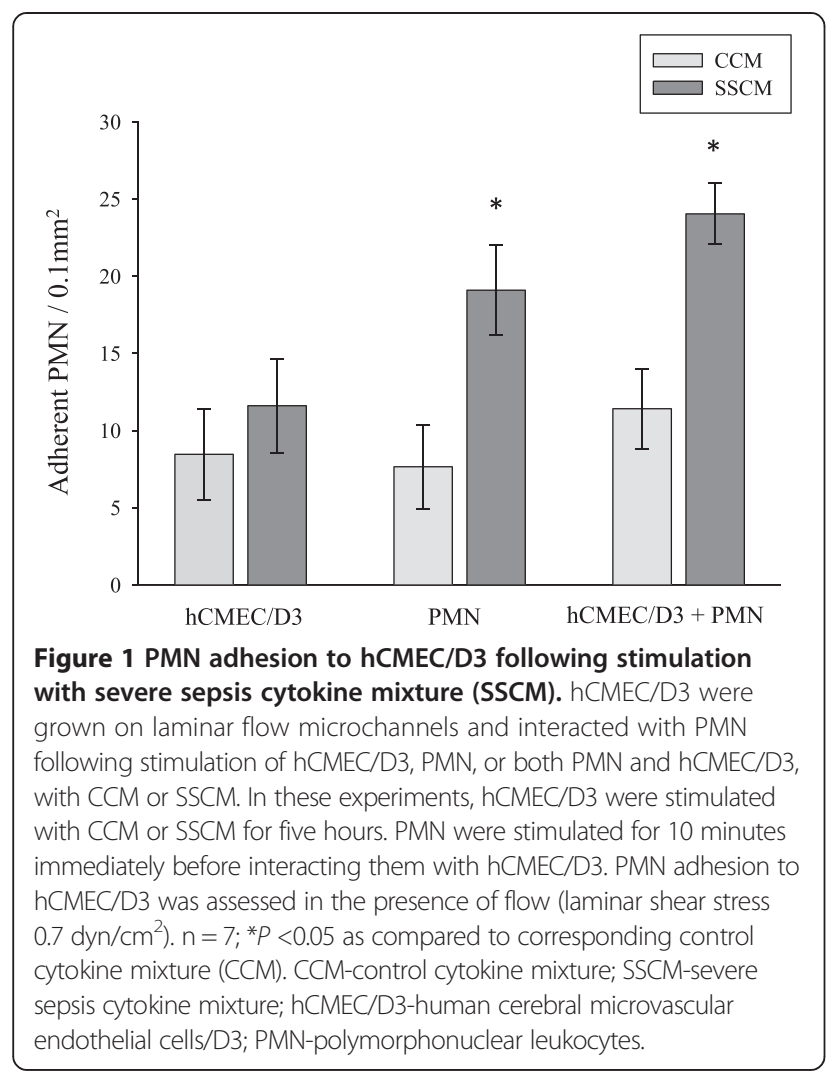

SSCM-stimulated PMN to SSCM-stimulated hCMEC/ D3 $(P<0.05 ; \mathrm{n}=6$; Figure 4$)$. The reduced PMN adhesion to hCMEC/D3 following immune-neutralization of ICAM-1 was likely due to interference with the constitutively expressed levels of ICAM-1 on the surface of hCMEC/D3, as SSCM failed to up-regulate ICAM-1 expression as assessed by qPCR (Figure 5).

\section{Discussion}

The present study identified an inflammatory profile in severe sepsis patients at ICU day one that up-regulated a pro-adhesive phenotype of PMN, resulting in increased adhesion to hCMEC/D3 in vitro. These effects may be important for the pathophysiology of SAE, as adhered and/or activated PMN could result in dysfunction of the BBB.

The extracellular environment of the brain is highly regulated by the BBB. Dysfunction of the BBB has been demonstrated with brain imaging in patients with $\mathrm{SAE}$ $[6,16,17]$, and $\mathrm{BBB}$ dysfunction is also observed in lipopolysaccharide-challenged animals [26]. Injury to the cellular components of the BBB, including cerebrovascular endothelial cells [27], pericytes [28], and astrocytes [29], was observed in experimental animal models of sepsis. Cerebrovascular endothelial cell dysfunction may lead to dysregulation of the brain extracellular environment

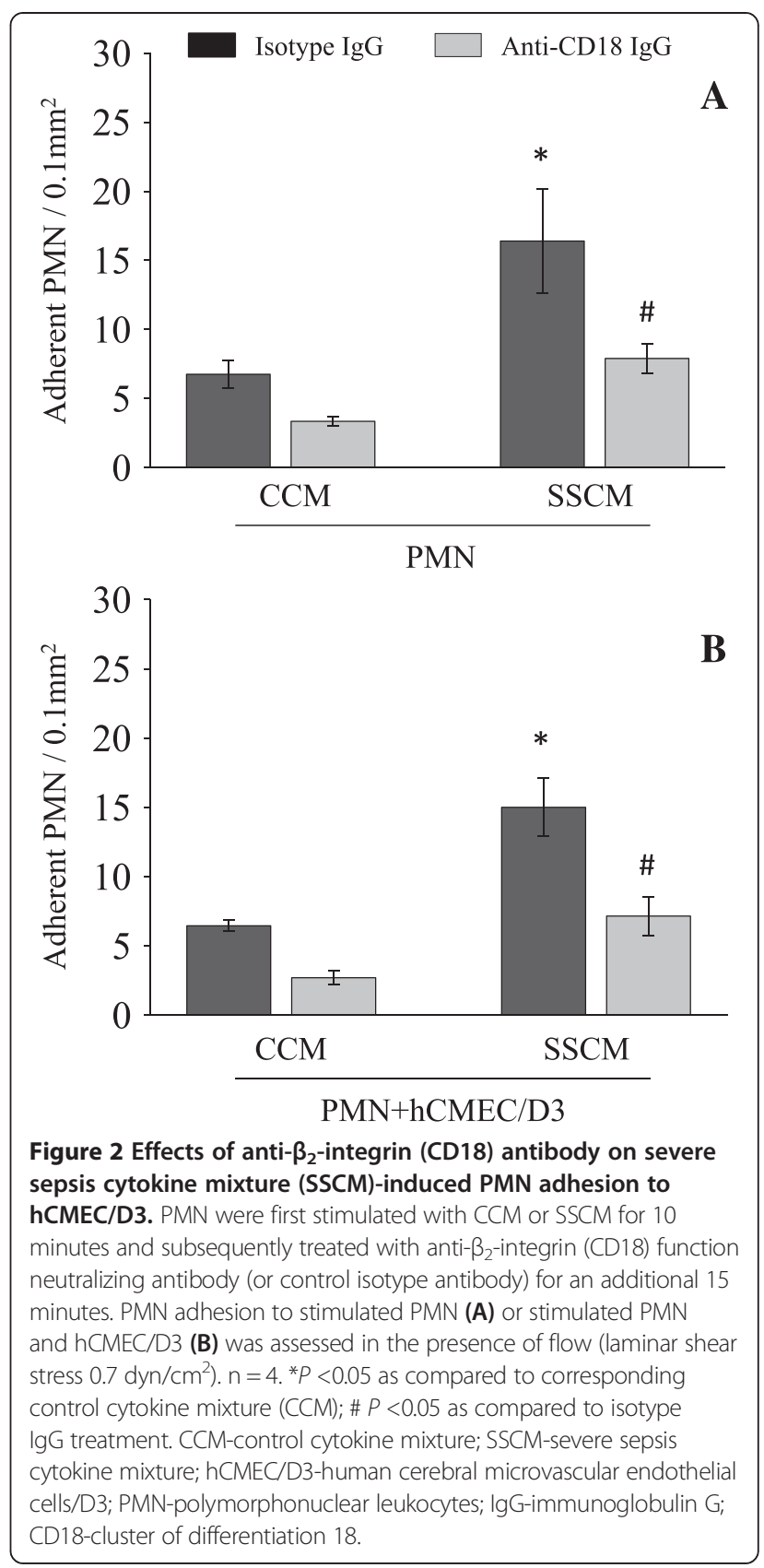

and subsequent neuronal dysfunction, thereby causing SAE.

In the current study, we collected blood plasma samples from ICU patients within the first 24 hours of severe sepsis diagnosis. Encephalopathy occurs early in sepsis, often developing on the first day of ICU admission [30]. Thus, blood was sampled at a critical time point for SAE development.

A total of 13 inflammatory analytes were up-regulated in blood plasma obtained from severe sepsis patients compared to blood plasma from age- and sex-matched healthy controls. Most of the up-regulated analytes are 


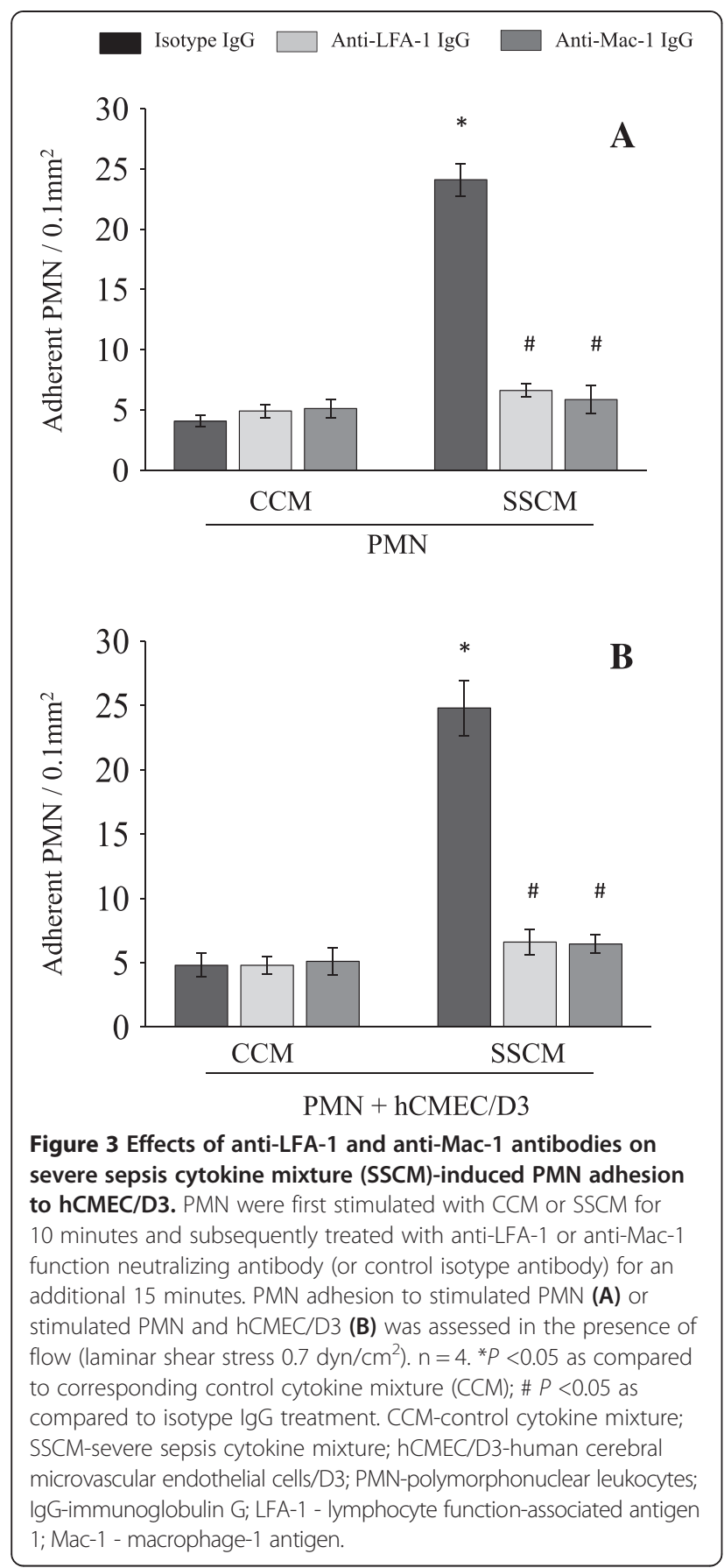

pro-inflammatory cytokines (such as IL-6) and CXC- and CC-family chemokines (such as IL-8, GRO- $\alpha$, MCP-1, and MIP-1 $\beta$ ), with well-documented roles in cellular activation and leukocyte recruitment to vascular endothelium $[31,32]$. In addition, a marked increase in cell growthmodulating factors (such as HGF, FGF $\beta$, HB-EGF, and KGF) in the circulation of severe sepsis patients was also found. The role of these growth factors in tissue remodelling has been shown previously, but the exact role of
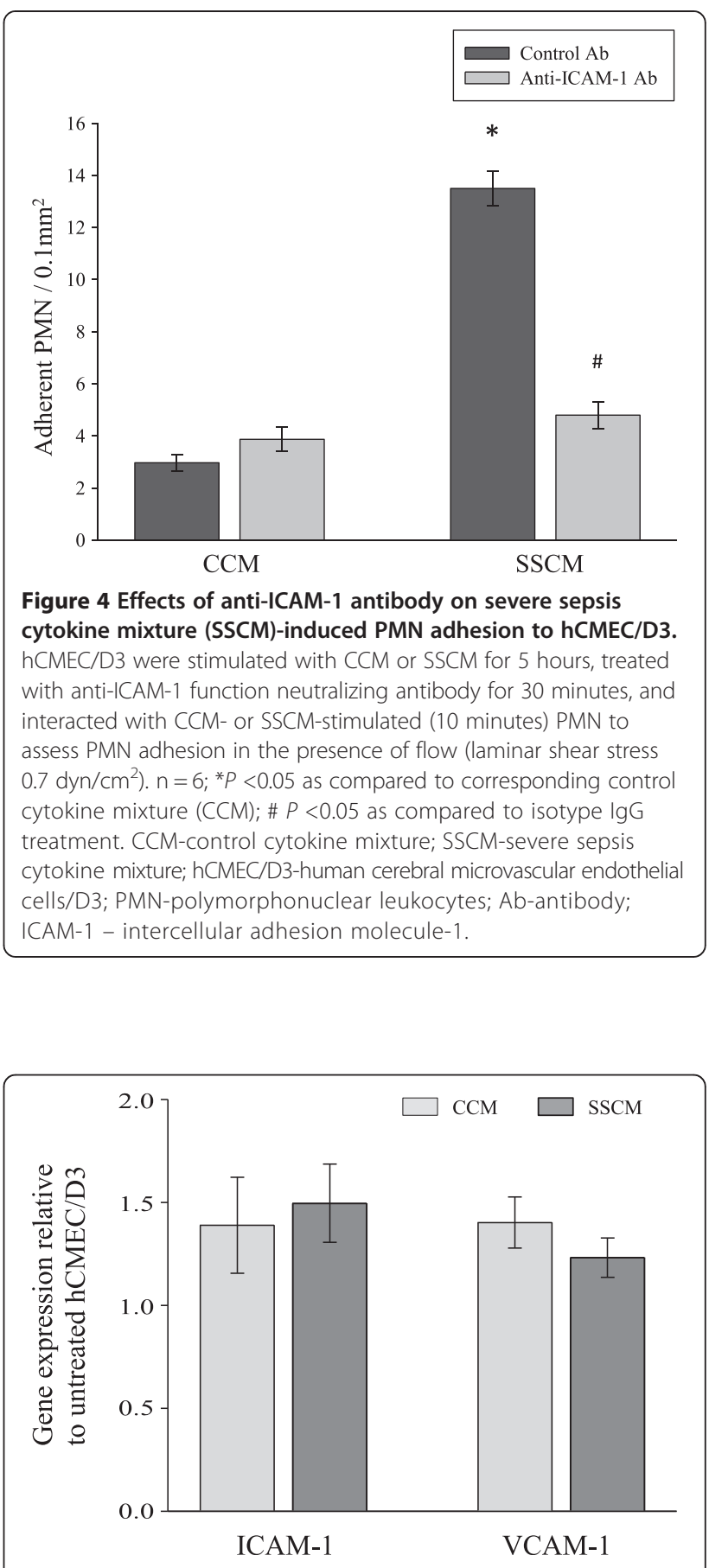

Figure 5 Effects of severe sepsis cytokine mixture (SSCM) on adhesion molecule ICAM-1 and VCAM-1 expression in hCMEC/D3. hCMEC/D3 were stimulated for 4 hours with either CCM or SSCM preceding analysis of VCAM-1 and ICAM-1 gene expression using qPCR. Data is presented as gene expression normalized to GAPDH and 185 expression, relative to $\mathrm{hCMEC/D3}$ treated with serum-free VascuLife EnGS-Mv cell culture media $(n=5)$. CCM-control cytokine mixture; SSCM-severe sepsis cytokine mixture; hCMEC/D3-human cerebral microvascular endothelial cells/D3; ICAM-1 - intercellular adhesion molecule-1; VCAM-1 - vascular cell adhesion molecule-1; 18S-18S ribosomal RNA; GAPDH-glyceraldehyde 3-phosphate dehydrogenase; qPCR- quantitative polymerase chain reaction. 
these molecules in the pathophysiology of severe sepsis is unknown.

Acute expression of individual cytokines and chemokines (such as IL-6, IL-8, MCP-1, and G-CSF) has been reported to correlate with the presence and severity of organ dysfunction in patients with severe sepsis and septic shock [22]. Interestingly, cytokines that are most commonly employed in experimental models of inflammation and/or sepsis (TNF- $\alpha$, IL- $1 \beta$, and IFN- $\gamma$ ) were not significantly increased in severe sepsis plasma obtained within 24 hours of ICU admission. One possible explanation for the latter phenomenon could be that the cytokines mentioned above may be expressed more prominently at different stages (for example, earlier or later than 24 hours post-admission time) and severities of sepsis, or in subgroups of severe sepsis patients [22,33]. Thus, it would be plausible to assume that the severe sepsis analyte profile within the first 24 hours of ICU admission represents not an 'early', but rather a 'later' cytokine response, that may intensify and perpetuate the inflammatory response.

One unique aspect of this study was the use of SSCM that consisted of eight cytokines and chemokines at the actual concentrations detected in the plasma of severe sepsis patients. In contrast, previous sepsis studies in the field have used a single cytokine and chemokine-based stimulation of the cells at often 'un-physiologically' high concentrations. In the current study we employed a severe sepsis analyte profile obtained at a time-point of disease that is clinically relevant to SAE development [3]. Our results indicate that PMN, but not hCMEC/D3, are more responsive to SSCM, with regards to up-regulation of the pro-adhesive phenotype. Stimulation of PMN, or PMN and hCMEC/D3, with SSCM resulted in an increased adhesion of PMN to hCMEC/D3 under in vitro conditions of flow, whereas stimulation of hCMEC/D3 only had no effect on PMN adhesion. This latter phenomenon may be explained by the inability of SSCM to up-regulate inducible adhesion molecule (such as ICAM-1 or VCAM-1) expression in hCMEC/D3 (as evidenced by qPCR analysis; Figure 5). On the other hand, the levels of constitutively expressed ICAM-1 on the surface of endothelial cells are sufficient to promote adhesion of activated PMN. Whether cerebrovascular endothelial cells (such as hCMEC/D3) are less sensitive to the pro-inflammatory cytokine and chemokine stimulation at physiologic concentrations as compared to the non-brain endothelial cells (such as pulmonary microvascular endothelial cells or umbilical vein endothelial cells) remains to be determined.

Our data indicate that $\beta_{2}$-integrins (such as LFA-1 and Mac-1) are required for SSCM-induced PMN adhesion to hCMEC/D3. Interestingly, immune-neutralization of ICAM-1 (a receptor for $\beta_{2}$-integrins) in hCMEC/D3 was also very effective in reducing PMN adhesion, despite the fact that SSCM failed to up-regulate ICAM-1 expression in hCMEC/D3. The latter may be explained by reduced availability of the constitutively expressed ICAM-1 following treatment of hCMEC/D3 with the immunoneutralizing anti-ICAM-1 antibody.

Up-regulated surface expression of $\beta_{2}$-integrin has been reported on freshly isolated PMN stimulated with blood plasma obtained from severe sepsis patients 24 hours after diagnosis [34]. Treatment of PMN with individual chemokines, such as IL-8, may also induce LFA-1- or Mac-1-dependent PMN adhesion [35,36]. The up-regulation of $\beta_{2}$-integrins (such as LFA-1 and Mac-1) surface expression is not always a prerequisite for LFA-1- and Mac-1-dependent adhesion. Rather, $\beta_{2}$-integrins that are constitutively expressed on the leukocyte cell membrane undergo conformational activation to a pro-adherent state that promotes leukocyte adhesion to molecules such as ICAM-1 [37,38]. LFA-1 and Mac-1 are known to bind ICAM-1 on vascular endothelial cells to facilitate PMN adhesion $[39,40]$. Ameliorated PMN recruitment to brain tissue has been demonstrated during endotoxemia in ICAM-1-deficient mice, similar to our findings in which an anti-ICAM-1 antibody reduced PMN adhesion to hCMEC/D3, suggesting an important role for $\beta_{2}$-integrin-ICAM-1 interactions in PMN adhesion to CMEC [41].

There is currently no data published regarding the use of antibody treatment directed against LFA-1, Mac-1, or $\beta_{2}$-integrin in humans for treatment of severe sepsis or SAE. However, LFA-1- and Mac-1-dependent recruitment of PMN is not unique to the brain, and studies that employed murine and rabbit models of sepsis have demonstrated that antibodies directed against $\beta_{2}$-integrin, LFA- 1 , and Mac-1 can reduce PMN recruitment and ameliorate subsequent dysfunction of lung and liver [42-44].

Whether PMN adhesion to the brain microvasculature in severe sepsis causes brain dysfunction is unclear. However, PMN are armed with many enzymes involved in host defense against pathogens, including NADPH oxidase, myeloperoxidase (MPO), matrix metalloproteinases (MMPs), elastase, and cathepsins [45]. Overwhelming local recruitment of activated PMN to brain microvasculature could expose CMEC to the deleterious effects of these enzymes. Both NADPH oxidase and MPO produce reactive oxygen species (ROS), which directly or indirectly contribute to the disruption of vascular endothelial cell intercellular junctions $[27,46,47]$. ROS production by PMN isolated from severe sepsis patients, and elevated MPO concentrations in blood plasma isolated from septic patients have both been reported $[11,48]$. MMPs and elastase can also damage endothelial cell adherens and tight junctions $[49,50]$. Disruption of the brain microvasculature may contribute to pathological activation of glial cells 
or neurons and, subsequently, to the development of SAE $[27,28]$.

At present, there is little pathophysiological data on SAE, and no studies using human tissues. Animal models of sepsis are a predominant source of data regarding potential mechanisms of brain dysfunction in sepsis. Excessive PMN recruitment, associated with neurological impairment in the brains of septic mice, has been reported $[13,51]$. PMN recruitment was ameliorated by treatment with antibodies directed against ICAM- $1, \beta_{2}$-integrin, and P-selectin adhesion molecules. Anti-P-selectin antibody treatment was associated with decreased sickness behaviour by septic mice, suggesting a possible role for PMNendothelial interactions in brain dysfunction during sepsis.

Our data have several limitations. First, our in vitro culture model may not fully represent the cerebral microvasculature in severe sepsis. Brain endothelial cells in vivo are in close contact with astrocytes, and co-culture of hCMEC/D3 with astrocytes has been demonstrated to decrease basal protein expression of ICAM-1 [52]. Therefore, hCMEC/D3 may express ICAM-1 at a higher basal rate compared to brain endothelial cells in vivo, which may reduce the window for up-regulation of ICAM-1 in response to cytokine stimulation. Second, our SSCM does not include all potential mediators that may play a role in leukocyte and/or endothelial activation in inflammation. The use of SSCM, however, provides valuable information regarding the acute inflammatory state in severe sepsis patients, at a time point when brain dysfunction most often occurs $[3,53]$.

\section{Conclusions}

Our data elucidate the late inflammatory changes in plasma during the first 24 hours of severe sepsis diagnosis in ICU patients, and demonstrate inflammation-induced PMN adhesion to hCMEC/D3, mediated by $\beta_{2}$-integrinICAM-1 interaction.

\section{Key messages}

- The pathophysiology of sepsis-associated encephalopathy (SAE) is unknown, but systemic inflammation may cause dysfunction of the brain microvasculature.

- We measured inflammatory analytes in plasma during severe sepsis, and created a severe sepsis cytokine mixture (SSCM).

- SSCM induced neutrophilic leukocyte (PMN) adhesion to cultured cerebrovascular endothelial cells in vitro.

- $\quad$ 2-integrins on PMNs, and ICAM-1 on cerebrovascular endothelial cells, mediated increased cellular adhesions.

- PMN adhesion to the brain microvasculature during severe sepsis may contribute to SAE.

\section{Abbreviations}

ACCP/SCCM: American College of Chest Physicians/Society of Critical Care Medicine; ANOVA: Analysis of variance; APACHE II: Acute Physiology and Chronic Health Evaluation II; BSA: bovine serum albumin; CCM: control cytokine mixture; CD: cluster of differentiation; CMEC: cerebral microvascular endothelial cells; EDTA: ethylenediaminetetraacetic acid; EGF: epidermal growth factor; EnGS-Mv: endothelial growth supplement-microvascular; FBS: fetal bovine serum; G-CSF: granulocyte colony-stimulating factor; GRO-a: Growth-regulated oncogene a; hCMEC/D3: human cerebral microvascular endothelial cells; hTERT: human telomerase reverse transcriptase; HGF: hepatocyte growth factor; ICAM-1: intercellular adhesion molecule 1; ICU: intensive care unit; IgG: immunoglobulin G; IL: interleukin; IP-10: interferon-inducible protein 10 kDa; LFA-1: lymphocyte function-associated antigen 1; Mac-1: macrophage-1 antigen; MCP-1: monocyte chemotactic protein-1; MIP-1 $\beta$ : macrophage inflammatory protein-1 beta; MMP: matrix metalloproteinase; MODS: multiorgan dysfunction syndrome; MPO: myeloperoxidase; NADPH: Nicotinamide adenine dinucleotide phosphate; PBS: phosphate buffered saline; PMN: polymorphonuclear leukocytes; qPCR: quantitative real-time polymerase chain reaction; ROS: reactive oxygen species; SAE: sepsis-associated encephalopathy; SSCM: severe sepsis cytokine mixture; SV40: Simian vacuolating virus 40.

\section{Competing interests}

The authors declare that they have no competing interests.

\section{Authors' contributions}

DDF, CMM, BY and GC initiated the study. PL collected patient data and blood samples. PYA, AO and DEC completed the antibody microarray in the laboratory of DDF. BW and GK provided antibody microarrays and analyzed the antibody microarray results. CB, BLD and EKP completed in vitro experiments in the laboratory of GC. CB, DDF and GC wrote the initial manuscript. All authors contributed revisions and approved the manuscript.

\section{Acknowledgements}

We would like to acknowledge Dr Pierre-Olivier Couraud (INSERM, Paris, France) for providing us with hCMEC/D3 cells. CB received graduate student funding from the Department of Pediatrics, Western University. DDF was supported by the grants from the Children's Health Foundation (London, ON, Canada) and the PSI Foundation. CMM received support from the Department of Medicine Program of Experimental Medicine (POEM). GC was supported by grants from the Heart and Stroke Foundation of Ontario (grant number: HSFO-393) and The Lawson Health Research Institute (grant number: IRF 25-12).

\section{Author details}

'Department of Physiology and Pharmacology, Western University, 1151 Richmond Str. North, London, ON N6A 5C1, Canada. ${ }^{2}$ Children's Health Research Institute, 800 Commissioners Road East, London, ON N6C 2V5, Canada. ${ }^{3}$ Centre for Critical IIIness Research, Lawson Health Research Institute, 800 Commissioners Rd East, London, ON N6C 6B5, Canada. ${ }^{4}$ Department of Paediatrics, Western University, 100 Collip Circle, London, ON N6G 4X8, Canada. ${ }^{5}$ Department of Clinical Neurological Sciences, Western University, 339 Windermere Road, London, ON N6A 5A5, Canada. ${ }^{6}$ Department of Medicine, Western University, 1151 Richmond Str. North, London, ON N6A 3K6, Canada. ${ }^{7}$ Department of Medicine, McMaster University, 1280 Main Street West, Hamilton, ON L8S 4K1, Canada. ${ }^{8}$ The Thrombosis and Atherosclerosis Research Institute, 237 Barton Str. East, Hamilton, ON L8L 2X2, Canada. ${ }^{9}$ Thermo Fisher Scientific, 3747 N Meridian Rd, Rockford, IL 61105, USA. ${ }^{10}$ London Regional Genomics Centre, Robarts Research Institute, 1151 Richmond Str. North, London, ON N6A 5B7, Canada. ${ }^{11}$ Department of Medical Biophysics, Western University, 1151 Richmond Str. North, London, ON N6A 5C1, Canada.

Received: 22 January 2015 Accepted: 13 March 2015 Published online: 07 April 2015

References

1. Angus DC, Linde-Zwirble WT, Lidicker J, Clermont G, Carcillo J, Pinsky MR. Epidemiology of severe sepsis in the United States: analysis of incidence, outcome, and associated costs of care. Crit Care Med. 2001;29:1303-10. 
2. Bone RC, Balk RA, Cerra FB, Dellinger RP, Fein AM, Knaus WA, et al. Definitions for sepsis and organ failure and guidelines for the use of innovative therapies in sepsis. The ACCP/SCCM Consensus Conference Committee. American College of Chest Physicians/Society of Critical Care Medicine. Chest. 1992;101:1644-55.

3. Young GB, Bolton CF, Austin TW, Archibald YM, Gonder J, Wells GA The encephalopathy associated with septic illness. Clin Invest Med. 1990;13:297-304

4. Sprung $\mathrm{CL}$, Peduzzi PN, Shatney $\mathrm{CH}$, Schein RM, Wilson MF, Sheagren JN et al. Impact of encephalopathy on mortality in the sepsis syndrome, The Veterans Administration Systemic Sepsis Cooperative Study Group. Crit Care Med. 1990;18:801-6.

5. Iwashyna TJ, Ely EW, Smith DM, Langa KM. Long-term cognitive impairment and functional disability among survivors of severe sepsis. JAMA. 2010;304:1787-94

6. Lamar CD, Hurley RA, Taber KH. Sepsis-associated encephalopathy: review of the neuropsychiatric manifestations and cognitive outcome. J Neuropsychiatry Clin Neurosci. 2011;23:237-41.

7. Brown KA, Brain SD, Pearson JD, Edgeworth JD, Lewis SM, Treacher DF. Neutrophils in development of multiple organ failure in sepsis. Lancet. 2006:368:157-69.

8. Mera S, Tatulescu D, Cismaru C, Bondor C, Slavcovici A, Zanc V, et al. Multiplex cytokine profiling in patients with sepsis. APMIS. 2011;119:155-63.

9. Ley K, Laudanna C, Cybulsky MI, Nourshargh S. Getting to the site of inflammation: the leukocyte adhesion cascade updated. Nat Rev Immunol. 2007;7:678-89.

10. Roberts AW. G-CSF: a key regulator of neutrophil production, but that's not all! Growth Factors. 2005:23:33-41.

11. Kothari N, Keshari RS, Bogra J, Kohli M, Abbas H, Malik A, et al. Increased myeloperoxidase enzyme activity in plasma is an indicator of inflammation and onset of sepsis. J Crit Care. 2011;26:435. e431-437.

12. van Griensven M, Probst C, Muller K, Hoevel P, Pape HC. Leukocyteendothelial interactions via ICAM-1 are detrimental in polymicrobial sepsis. Shock. 2006;25:254-9.

13. Vachharajani V, Vital S, Russell J, Granger DN. Hypertonic saline and the cerebral microcirculation in obese septic mice. Microcirculation. 2007;14:223-31.

14. Gavins FN, Hughes EL, Buss NA, Holloway PM, Getting SJ, Buckingham JC. Leukocyte recruitment in the brain in sepsis: involvement of the annexin 1-FPR2/ALX anti-inflammatory system. FASEB J. 2012;26:4977-89.

15. Schmidt EP, Lee WL, Zemans RL, Yamashita C, Downey GP. On, around, and through: neutrophil-endothelial interactions in innate immunity. Physiology (Bethesda). 2011;26:334-47.

16. Piazza O, Cotena S, De Robertis E, Caranci F, Tufano R. Sepsis associated encephalopathy studied by MRI and cerebral spinal fluid S100B measurement. Neurochem Res. 2009;34:1289-92.

17. Finelli PF, Uphoff DF. Magnetic resonance imaging abnormalities with septic encephalopathy. J Neurol Neurosurg Psychiatry. 2004;75:1189-91.

18. Yazdan-Ashoori P, Liaw P, Toltl L, Webb B, Kilmer G, Carter DE, et al. Elevated plasma matrix metalloproteinases and their tissue inhibitors in patients with severe sepsis. J Crit Care. 2011;26:556-65.

19. Bernard GR, Ely EW, Wright TJ, Fraiz J, Stasek Jr JE, Russell JA, et al. Safety and dose relationship of recombinant human activated protein $C$ for coagulopathy in severe sepsis. Crit Care Med. 2001;29:2051-9.

20. Garcia 2nd BH, Hargrave A, Morgan A, Kilmer G, Hommema E, Nahrahari J, et al. Antibody microarray analysis of inflammatory mediator release by human leukemia T-cells and human non small cell lung cancer cells. J Biomol Tech. 2007:18:245-51.

21. Casey LC, Balk RA, Bone RC. Plasma cytokine and endotoxin levels correlate with survival in patients with the sepsis syndrome. Ann Intern Med. 1993;119:771-8.

22. Bozza FA, Salluh Jl, Japiassu AM, Soares M, Assis EF, Gomes RN, et al. Cytokine profiles as markers of disease severity in sepsis: a multiplex analysis. Crit Care. 2007;11:R49.

23. Weksler BB, Subileau EA, Perriere N, Charneau P, Holloway K, Leveque M, et al. Blood-brain barrier-specific properties of a human adult brain endothelial cell line. FASEB J. 2005;19:1872-4.

24. Omatsu T, Cepinskas G, Clarson C, Patterson EK, Alharfi IM, Summers K, et al. CXCL1/CXCL8 (GROalpha/IL-8) in human diabetic ketoacidosis plasma facilitates leukocyte recruitment to cerebrovascular endothelium in vitro. Am J Physiol Endocrinol Metab. 2014;306:E1077-84.
25. Serizawa F, Patterson E, Potter RF, Fraser DD, Cepinskas G. Pre-treatment of human cerebrovascular endothelial cells with CO-releasing molecule-3 (CORM-3) interferes with JNKVAP-1 signaling and suppresses LPS-induced pro-adhesive phenotype. Microcirculation. 2015:22:28-36.

26. Alexander JJ, Jacob A, Cunningham P, Hensley L, Quigg RJ. TNF is a key mediator of septic encephalopathy acting through its receptor, TNF receptor-1. Neurochem Int. 2008;52:447-56.

27. Handa O, Stephen J, Cepinskas G. Role of endothelial nitric oxide synthase-derived nitric oxide in activation and dysfunction of cerebrovascular endothelial cells during early onsets of sepsis. Am J Physiol Heart Circ Physiol. 2008;295:H1712-9.

28. Nishioku T, Dohgu S, Takata F, Eto T, Ishikawa N, Kodama KB, et al. Detachment of brain pericytes from the basal lamina is involved in disruption of the blood-brain barrier caused by lipopolysaccharide-induced sepsis in mice. Cell Mol Neurobiol. 2009;29:309-16.

29. Papadopoulos MC, Lamb FJ, Moss RF, Davies DC, Tighe D, Bennett ED. Faecal peritonitis causes oedema and neuronal injury in pig cerebral cortex. Clin Sci. 1999:96:461-6

30. Wilson JX, Young GB. Progress in clinical neurosciences: sepsis-associated encephalopathy: evolving concepts. Can J Neuro Sci. 2003;30:98-105.

31. Cloutier A, Ear T, Blais-Charron E, Dubois CM, McDonald PP. Differential involvement of NF-kappaB and MAP kinase pathways in the generation of inflammatory cytokines by human neutrophils. J Leukoc Biol. 2007;81:567-77.

32. Moser B, Loetscher M, Piali L, Loetscher P. Lymphocyte responses to chemokines. Int Rev Immunol. 1998;16:323-44.

33. Castellheim A, Thorgersen EB, Hellerud BC, Pharo A, Johansen HT, Brosstad $F$, et al. New biomarkers in an acute model of live Escherichia coli-induced sepsis in pigs. Scand J Immunol. 2008;68:75-84.

34. Horner C, Schuster S, Plachky J, Hofer S, Martin E, Weigand MA. Hemofiltration and immune response in severe sepsis. J Surg Res. 2007;142:59-65.

35. Takami M, Terry $\vee$, Petruzzelli L. Signaling pathways involved in IL-8-dependent activation of adhesion through Mac-1. J Immunol. 2002;168:4559-66.

36. Lomakina EB, Waugh RE. Signaling and dynamics of activation of LFA-1 and Mac-1 by immobilized IL-8. Cell Mol Bioeng. 2010;3:106-16.

37. Diamond MS, Springer TA. The dynamic regulation of integrin adhesiveness. Current Biol. 1994;4:506-17.

38. de Vries WR, Bernards NT, de Rooij MH, Koppeschaar HP. Dynamic exercise discloses different time-related responses in stress hormones. Psychosom Med. 2000;62:866-72.

39. Bunge MA, Mahner M. Scientific realism: selected essays of Mario Bunge. Amherst, NY: Prometheus Books; 2001

40. Smith CW, Marlin SD, Rothlein R, Toman C, Anderson DC. Cooperative interactions of LFA-1 and Mac-1 with intercellular adhesion molecule-1 in facilitating adherence and transendothelial migration of human neutrophils in vitro. J Clin Invest. 1989;83:2008-17

41. Bohatschek M, Werner A, Raivich G. Systemic LPS injection leads to granulocyte influx into normal and injured brain: effects of ICAM-1 deficiency. Exp Neurol. 2001;172:137-52.

42. Wong KF, Wo J, Ho D, Poon RT, Casasnovas JM, Luk JM. Prophylactic uses of integrin CD18-betaA peptide in a murine polymicrobial peritonitis model. World J Gastroenterol. 2010;16:2648-56.

43. Asaduzzaman M, Zhang S, Lavasani S, Wang Y, Thorlacius H. LFA-1 and MAC-1 mediate pulmonary recruitment of neutrophils and tissue damage in abdominal sepsis. Shock. 2008;30:254-9.

44. Gardinali M, Borrelli E, Chiara O, Lundberg C, Padalino P, Conciato L, et al Inhibition of CD11-CD18 complex prevents acute lung injury and reduces mortality after peritonitis in rabbits. Am J Respir Crit Care Med. 2000;161:1022-9.

45. Smith JA. Neutrophils, host defense, and inflammation: a double-edged sword. J Leukoc Biol. 1994;56:672-86.

46. Schreibelt G, Kooij G, Reijerkerk A, van Doorn R, Gringhuis SI, van der Pol S, et al. Reactive oxygen species alter brain endothelial tight junction dynamics via RhoA, PI3 kinase, and PKB signaling. FASEB J. 2007;21:3666-76.

47. Patterson EK, Fraser DD, Capretta A, Potter RF, Cepinskas G. Carbon monoxide-releasing molecule 3 inhibits myeloperoxidase (MPO) and protects against MPO-induced vascular endothelial cell activation/dysfunction. Free Radic Biol Med. 2014;70:167-73.

48. Martins PS, Kallas EG, Neto MC, Dalboni MA, Blecher S, Salomao R. Upregulation of reactive oxygen species generation and phagocytosis, and 
increased apoptosis in human neutrophils during severe sepsis and septic shock. Shock. 2003;20:208-12.

49. Yang Y, Estrada EY, Thompson JF, Liu W, Rosenberg GA. Matrix metalloproteinase-mediated disruption of tight junction proteins in cerebral vessels is reversed by synthetic matrix metalloproteinase inhibitor in focal ischemia in rat. J Cereb Blood Flow Metab. 2007;27:697-709.

50. Ionescu CV, Cepinskas G, Savickiene J, Sandig M, Kvietys PR. Neutrophils induce sequential focal changes in endothelial adherens junction components: role of elastase. Microcirculation. 2003;10:205-20.

51. Vachharajani V, Russell JM, Scott KL, Conrad S, Stokes KY, Tallam L, et al. Obesity exacerbates sepsis-induced inflammation and microvascular dysfunction in mouse brain. Microcirculation. 2005;12:183-94.

52. Urich E, Lazic SE, Molnos J, Wells I, Freskgard PO. Transcriptional profiling of human brain endothelial cells reveals key properties crucial for predictive in vitro blood-brain barrier models. PLoS One. 2012;7, e38149.

53. Rivers E, Nguyen B, Havstad S, Ressler J, Muzzin A, Knoblich B, et al. Early goal-directed therapy in the treatment of severe sepsis and septic shock N Engl J Med. 2001;345:1368-77.

\section{Submit your next manuscript to BioMed Central and take full advantage of:}

- Convenient online submission

- Thorough peer review

- No space constraints or color figure charges

- Immediate publication on acceptance

- Inclusion in PubMed, CAS, Scopus and Google Scholar

- Research which is freely available for redistribution 